\title{
INVOLUTIONS ON FINITE-DIMENSIONAL ALGEBRAS OVER REAL CLOSED FIELDS
}

\author{
W. D. MUNN \\ (Received 29 August 2002; revised 21 July 2003) \\ Communicated by D. Easdown
}

\begin{abstract}
It is shown that the following conditions on a finite-dimensional algebra $A$ over a real closed field or an algebraically closed field of characteristic zero are equivalent: (i) $A$ admits a special involution, in the sense of Easdown and Munn, (ii) $A$ admits a proper involution, (iii) $A$ is semisimple.
\end{abstract}

2000 Mathematics subject classification: primary $16 \mathrm{~W} 10$.

\section{Introduction}

A field is termed formally real if -1 cannot be expressed in it as a sum of squares and real closed if it is a formally real field that has no formally real proper algebraic extension. Many real closed fields exist; examples include the real field $\mathbb{R}$ and the field of all real algebraic numbers.

It is clear that a real closed field $F$ has characteristic zero. Less obvious is the fact that $F$ is totally ordered by the rule that $a \leq b$ if and only if $b-a=c^{2}$ for some $c$ [5, Section 70, Theorem 1]. We shall make use of this total ordering without further comment.

Recall that an involution on a ring $R$ is a mapping * $: R \rightarrow R$ such that

$$
(\forall a, b \in R) \quad(a+b)^{*}=a^{*}+b^{*}, \quad(a b)^{*}=b^{*} a^{*}, \quad a^{* *}=a .
$$

Now let $A$ be an algebra over a real closed field $F$. An involution on $A$ is defined to be an involution * on the ring $(A,+, \cdot)$ with the additional property that

$$
(\forall a \in A)(\forall \lambda \in F) \quad(\lambda a)^{*}=\lambda a^{*} .
$$

(C) 2004 Australian Mathematical Society $1446-7887 / 04 \$ A 2.00+0.00$ 
Two types of involution * on $A$ concern us here. We say that ${ }^{*}$ is

(i) proper if $a a^{*}=0$ implies $a=0$ for all $a \in A$,

(ii) special if, for every nonempty finite subset $T$ of $A$,

$$
(\exists t \in T)(\forall u, v \in T) \quad t t^{*}=u v^{*} \Rightarrow u=v .
$$

Note that, in each case, the defining condition is on the multiplicative semigroup of $A$. The notion of a special involution was introduced in [2]. It is perhaps surprising that many naturally occurring involutions are special: for instance, transposition on the algebra of all real $n \times n$ matrices and conjugation on the algebra of all real quaternions [2], the mapping

$$
\sum_{x \in S} \alpha_{x} x \mapsto \sum_{x \in S} \alpha_{x} x^{-1}
$$

on the semigroup algebra $\mathbb{R}[S]$ of an inverse semigroup $S$ over $\mathbb{R}$ (in particular, on the group algebra $\mathbb{R}[S]$, where $S$ is a group) [3], and the mapping

$$
\sum_{w \in M} \alpha_{w} w \mapsto \sum_{w \in M} \alpha_{w} \overleftarrow{w}
$$

on $\mathbb{R}[M]$, where $M$ is a free monoid of arbitrary rank and $\overleftarrow{w}$ denotes the reverse of the word $w$ in $M$ [1].

The two properties are not independent: in fact, every special involution is proper, as we now show. Let * be a special involution on an algebra $A$ over a real closed field and let $a \in A$ be such that $a a^{*}=0$. Take $T:=\{a, 0\}$. Then there exists $t \in T$ such that, for all $u, v \in T, t t^{*}=u v^{*}$ implies $u=v$. For each possibility, $t t^{*}=0=0 a^{*}$ and so $a=0$. Thus ${ }^{*}$ is proper. However, a simple example demonstrates that not every proper involution is special. Let $A$ denote the group algebra $\mathbb{R}[G]$, where $G$ is the cyclic group of order 4 . Since $A$ is commutative, the identity mapping on $A$ is an involution; moreover, since $A$ is semisimple then, for $a \in A, a^{2}=0$ implies $a=0$. Thus the identity mapping is proper. However, if $g$ denotes a generator of $G$ then, by taking $T:=G$ and noting the equations $\left(g^{0}\right)^{2}=g g^{3}=\left(g^{2}\right)^{2}$ and $g^{2}=g^{0} g^{2}=\left(g^{3}\right)^{2}$, we see that the identity mapping is not special.

The aim of the present paper is to show that, on a finite-dimensional algebra $A$ over a real closed field, the following conditions are equivalent: (i) $A$ admits a special involution, (ii) $A$ admits a proper involution, (iii) $A$ is semisimple. (In the previous paragraph, we have an example of an involution on a real finite-dimensional semisimple algebra that is proper but not special; however, a different involutionnamely that induced by inversion in the group — is special.) With a natural adjustment to the definition of an involution, a similar result follows for a finite-dimensional algebra over an algebraically closed field of characteristic zero. These results, for the 
real and complex fields, were announced in [4]. In each case, the author's proof used a result in representation theory. The direct proofs given here are extensions of the argument in [2, Example 4].

\section{Finite-dimensional algebras over real closed fields}

We begin by considering division algebras. A classical theorem of Frobenius states that, to within isomorphism, the only finite-dimensional division algebras over $\mathbb{R}$ are $\mathbb{R}$ itself, the complex field $\mathbb{C}$ and the algebra $\mathbb{H}$ of real quaternions [5, Section 131, pages 201-202]. The proof of this theorem applies also to the case where $\mathbb{R}$ is replaced by any real closed field $F$, for it depends only on two particular properties [5, Section 70, Theorems 1 and 3]:

(i) $F$ is totally ordered and contains a square root of each non-negative element,

(ii) the field obtained from $F$ by adjoining a root of the irreducible polynomial $x^{2}+1$ is algebraically closed.

All the details of the lemma below now follow routinely.

LEMMA 2.1. Every finite-dimensional division algebra $D$ over a real closed field $F$ admits an involution ${ }^{c}: D \rightarrow D, d \mapsto d^{c}$ ('conjugation') such that

$$
(\forall d \in D) \quad d d^{c} \in F, \quad d d^{c} \geq 0, \quad d d^{c}=0 \Rightarrow d=0,
$$

according to the cases

(i) $D=F: \alpha^{c}=\alpha(\alpha \in F)$;

(ii) $D=F[i]$, where $i^{2}=-1:(\alpha+\beta i)^{c}=\alpha-\beta i(\alpha, \beta \in F)$;

(iii) $D=F[i, j, k]$, where $i^{2}=j^{2}=k^{2}=-1, i j=k=-j i, j k=i=-k j$; $k i=j=-i k$ :

$$
(\alpha+\beta i+\gamma j+\delta k)^{c}=\alpha-\beta i-\gamma j-\delta k \quad(\alpha, \beta, \gamma, \delta \in F) .
$$

Observe that the lemma states, in particular, that every finite-dimensional division algebra over a real closed field admits a proper involution.

THEOREM 2.2. The following conditions on a finite-dimensional algebra $A$ over a real closed field are equivalent:

(i) A admits a special involution,

(ii) A admits a proper involution,

(iii) A is semisimple.

PROOF. Since, as remarked in Section 1, every special involution is a proper involution, (i) implies (ii). 
A standard short argument, which we include for completeness, shows that (ii) implies (iii). Let ${ }^{*}$ be a proper involution on $A$ and let $N$ denote the radical of $A$. Suppose that $a \in N \backslash 0$. Then $a a^{*} \in N$ and so $\left(a a^{*}\right)^{m}=0$ for some least positive integer $m$. Since ${ }^{*}$ is proper, $m \geq 2$. Write $b:=\left(a a^{*}\right)^{m-1}$. Then $b=b^{*}$ and so $b b^{*}=b^{2}=\left(a a^{*}\right)^{2 m-2}=0$, since $2 m-2 \geq m$. Thus $b=0$, contrary to the minimality of $m$. Hence $N=0$ and so $A$ is semisimple.

We complete the proof by showing that (iii) implies (i). Denote the ground field of $A$ by $F$. Consider first the algebra $M_{n}(D)$ of all $n \times n$ matrices over a finitedimensional division algebra $D$ over $F$. Define ${ }^{\dagger}: M_{n}(D) \rightarrow M_{n}(D), a \mapsto a^{\dagger}$, by writing $a^{\dagger}:=\left(a^{c}\right)^{T}$, where $a^{c}$ denotes the matrix obtained from $a$ by replacing each entry $a_{i j}$ by $a_{i j}{ }^{c}$ (with ${ }^{c}$ as in Lemma 2.1) and ${ }^{T}$ denotes transposition. Since ${ }^{c}$ is an involution on $D$, it follows easily that ${ }^{\dagger}$ is an involution on $M_{n}(D)$. Now denote the trace of $a \in M_{n}(D)$ by $\tau(a)$. Then, for all $a=\left[a_{i j}\right] \in M_{n}(D)$, we have that $\tau\left(a a^{\dagger}\right)=\sum_{i, j=1}^{n} a_{i j} a_{i j} c$ and so, by Lemma 2.1 ,

$$
\left(\forall a \in M_{n}(D)\right) \quad \tau\left(a a^{\dagger}\right) \in F, \quad \tau\left(a a^{\dagger}\right) \geq 0, \quad \tau\left(a a^{\dagger}\right)=0 \Rightarrow a=0 .
$$

Let $A$ be semisimple. By Wedderburn's theorem, we may assume, without loss of generality, that $A$ is the external direct sum of algebras $A_{i}(i=1, \ldots, k)$, where, for each $i, A_{i}=M_{n_{i}}\left(D_{i}\right)$ for some positive integer $n_{i}$ and some finite-dimensional division algebra $D_{i}$ over $F$. No confusion should arise from the use of the same symbol ${ }^{\dagger}$ to denote the involution defined on each $M_{n_{i}}\left(D_{i}\right)$ as in the previous paragraph. For all $a \in A$, denote the $A_{i}$-component of $a$ by $a_{i}(i=1, \ldots, k)$. Then ${ }^{*}: A \rightarrow A, a \mapsto a^{*}$ defined by $\left(a^{*}\right)_{i}=a_{i}{ }^{\dagger}(i=1, \ldots, k)$ is readily seen to be an involution on $A$. We show that it is special.

Let $T$ be a nonempty finite subset of $A$. Choose $t \in T$ such that

$$
\sum_{i=1}^{k} \tau\left(t_{i} t_{i}^{\dagger}\right)=\max \left\{\sum_{i=1}^{k} \tau\left(w_{i} w_{i}^{\dagger}\right): w \in T\right\} .
$$

Suppose that $t t^{*}=u v^{*}$, for some $u, v \in T$. Then, for each $i$,

$$
t_{i} t_{i}^{\dagger}=u_{i} v_{i}^{\dagger}=\left(u_{i} v_{i}^{\dagger}\right)^{\dagger}=v_{i} u_{i}^{\dagger}
$$

and so

$$
\left(u_{i}-v_{i}\right)\left(u_{i}-v_{i}\right)^{\dagger}=u_{i} u_{i}^{\dagger}+v_{i} v_{i}^{\dagger}-2 t_{i} t_{i}^{\dagger} .
$$

Thus, by (1) and (2),

$$
\begin{aligned}
0 & \leq \sum_{i=1}^{k} \tau\left(\left(u_{i}-v_{i}\right)\left(u_{i}-v_{i}\right)^{\dagger}\right) \\
& =\sum_{i=1}^{k} \tau\left(u_{i} u_{i}^{\dagger}\right)+\sum_{i=1}^{k} \tau\left(v_{i} v_{i}^{\dagger}\right)-2 \sum_{i=1}^{k} \tau\left(t_{i} t_{i}^{\dagger}\right) \leq 0 .
\end{aligned}
$$


Hence $\sum_{i=1}^{k} \tau\left(\left(u_{i}-v_{i}\right)\left(u_{i}-v_{i}\right)^{\dagger}\right)=0$. Thus, by (1), $u_{i}=v_{i}$ for each $i$. Consequently, $u=v$. This shows that ${ }^{*}$ is special.

An analogous result (Corollary 2.3) holds for algebras over algebraically closed fields of characteristic zero. As noted earlier, if we adjoin to a real closed field a root of $x^{2}+1$ then the resulting field is algebraically closed. In fact, all algebraically closed fields arise in this way. A statement, with a proof for the countable case, is given in [5, Section 71]; the general case is a simple application of Zorn's lemma. Let $A$ be an algebra over an algebraically closed field $F$ of characteristic zero. Then $F=R[i]$, where $R$ is a maximal formally real subfield and $i^{2}=-1$. Define ' ('conjugation') on $F$ by taking $(\xi+\eta i)^{r}=\xi-\eta i(\xi, \eta \in R)$. By an involution ${ }^{*}$ on $A$ we now mean a ring involution such that

$$
(\forall a \in A)(\forall \lambda \in F) \quad(\lambda a)^{*}=\lambda^{c} a^{*} .
$$

Proper and special involutions are defined as before. Suppose that $A$ is semisimple. Since $F$ is algebraically closed, the only finite-dimensional division algebra over $F$ is $F$ itself and so $A$ is isomorphic to a direct sum of full matrix algebras over $F$. Now regard $A$ as an algebra over $R$. Then ${ }^{*}: A \rightarrow A$, constructed from ${ }^{c}$ as in the proof that (iii) implies (i) in Theorem 2.2, is a special involution; moreover, it satisfies (3). Hence ${ }^{*}$ is a special involution on $A$ as an algebra over $F$. Thus we obtain the result below:

COROLLARY 2.3. The following conditions on a finite-dimensional algebra A over an algebraically closed field of characteristic zero are equivalent: $A$ admits a special involution, $A$ admits a proper involution, $A$ is semisimple.

\section{Acknowledgement}

I am grateful to the referee for several very helpful suggestions.

\section{References}

[1] M. J. Crabb, C. M. McGregor, W. D. Munn and S. Wassermann, 'On the algebra of a free monoid', Proc. Roy. Soc. Edinburgh Sect. A 126 (1996), 939-945.

[2] D. Easdown and W. D. Munn, 'On semigroups with involution', Bull. Austral. Math. Soc. 48 (1993), 103-110.

[3] __. 'Trace functions on inverse semigroup algebras', Bull. Austral. Math. Soc. 52 (1995), 359372. 
[4] W. D. Munn, 'Special involutions', in: Semigroup theory and its applications (New Orleans, LA, 1994) (eds. K. H. Hofmann and M. W. Mislove), London Math. Soc. Lecture Note Ser. 231 (Cambridge University Press, Cambridge, 1996) pp. 157-165.

[5] B. L. Van der Waerden, Modern algebra, English edition (Ungar, New York, 1949 (Vol. I) and 1950 (Vol. II)).

\section{Department of Mathematics}

University of Glasgow

Glasgow G12 8QW

Scotland

UK

e-mail: wdm@maths.gla.ac.uk 